\title{
Identification and Biotyping of Escherichia coli from Diarrheic Lambs in and Around Debre Birhan Town, Ethiopia
}

Metasebia Aklilu $^{1 *}$, Tesfaye Sisay ${ }^{2}$, Genene Tefera ${ }^{3}$ and Belay Tekalign ${ }^{4}$

${ }^{1}$ Addis Ababa University, College of Veterinary Medicine and Agriculture, Debrezeite, Ethiopia

${ }^{2}$ Addis Ababa University, Institute Biotechnology, Department of Health Biotechnology, Addis Ababa, Ethiopia

${ }^{3}$ Institute of Biodiversity center, Addis Ababa, Ethiopia

${ }^{4}$ Mereced University, California, USA

\begin{abstract}
Background: Infectious diarrhea is the most significant cause of morbidity and mortality in neonatal dairy sheep throughout the world. This is the first study conducted on isolation and biotyping of $E$. coli from lamb diarrhea in and around Debre Birhan. The present study was undertaken with the objective of isolation, omnilog characterization and biotyping of $E$. coli isolates from faecal samples around Debrebrhan.

Methods: A cross-sectional study was conducted from October, 2012 to April, 2013. The study focused on lambs less than three months of age showing clinical symptoms of diarrhea. Standard cultural and Omnilog tests were done to identify E. coli species and biotypes; descriptive statistics and Chi-square were used to analyze the collected data.

Results: From a total of 100 diarrheic lambs examined, $84 \%$ were found to be positive for $E$. coli. On the basis of fermentation reactions of sugars viz. dulcitol, raffinose, rhamnose, salicin, starch and sucrose, 70 isolates utilized one or more sugar while 14 isolates failed to utilize any of the sugars. $70 \mathrm{E}$. coli isolates were biotyped into 15 different combinations. Association between different age groups and occurrence of lamb diarrhea caused by $E$. coli strains showed a significant association $(p<0.05)$. From the questionnaire findings, $60 \%$ of all the health problems in lambs were due to diarrhea where young sheep were more affected.

Conclusion: pathogenic $E$. coli has the potential to cause sheep diarrhea. The distribution of the $E$. coli isolates in to different biotypes indicates the diverse nature of the organism. Therefore, further detailed study should be carried out to understand the role of $E$. coli in lamb diarrhea and identify the virulent strains involved.
\end{abstract}

Keywords: Biotyping; Debre Birhan; Lamb diarrhea; Omnilog; Sugars; Ethiopia

\section{Introduction}

E. coli is a multitalented, enteric Gram-negative bacillus, and best known as a noninvasive commensal that grows in mass culture in human and in animal gut lumen, perhaps keeping other more harmful bacteria away from proliferating [1]. Diarrhea is common in newborn calves, lambs, and kids. The acute disease is characterized by progressive dehydration and death, sometimes in as few as $12 \mathrm{hrs}$. In the subacute form, diarrhea may persist for several days and result in malnutrition and emaciation. Lambs are vulnerable to $E$. coli infection. Two age groups appear to be susceptible, lambs of 1-2 days of age and lambs of 3-8 weeks old. Symptoms include diarrhea, a rise in temperature, weakness and lack of appetite. This is soon followed by coma and death within a few hours. In older animals there is a tendency or infection to localize itself in the joints of survivors. Lesions include enlarged, haemorrhagic spleens, and the accumulation of synovial fluid and sometimes pus in affected joints [2].

The E. coli infection is a disease of economic importance. The mortality rate due to $E$. coli infection in sheep ranged from $1-5 \%$ with an age distribution of 3-12 weeks old. Due to E. coli infection in sheep wool and meat production declined dramatically. As a result the farmers who are economically dependent on sheep rearing become looser [3].

Cultural characterization of $E$. coli by using different media and biochemical characterization by observing variable reaction to different sugars and chemicals are the basic rules for their identification. Antibiotics are widely used in case of diseased animal in the treatment of Sheep diarrhea [4].
Knowledge of local antimicrobial therapy pattern is important in selecting the appropriate therapy. Various parameters including the prevalence, isolation, identification and epidemiological investigation of $E$. coli was studied by various workers [5]. Zinnah [5], conducted an experiment on E. coli isolated from human, cattle, sheep, goat, chickens, duck, pigeon, drain sewages and soil. For the prevention and control of any microbial disease, prior isolation, identification and characterization of that particular etiological agent in a country is a prerequisite.

E. coli are able to ferment a variety of carbohydrate substrates, generally by converting them to glucose or to a substrate on the fermentative chain of the breakdown of glucose. The ability to ferment a given sugar of the types described above by a strain of E.coli is dependent on the strain having the requisite enzymes to convert it to glucose or to a substance on the degradative chain from glucose [6].

Intestinal lesions caused by AEEC are termed attaching and

*Corresponding author: Metasebia Aklilu, Department of Microbiology Immunology and Veterinary Public Health, College of Veterinary Medicine and Agriculture, Addis Ababa University, Addis Ababa, Ethiopia, E-mail: aklilumetasebia@gmail.com

Received July 25, 2013; Accepted August 29, 2013; Published September 02 2013

Citation: Aklilu M, Sisay T, Tefera G, Tekalign B (2013) Identification and Biotyping of Escherichia coli from Diarrheic Lambs in and Around Debre Birhan Town Ethiopia. J Environ Anal Toxicol 3: 188. doi:10.4172/2161-0525.1000188

Copyright: $\odot 2013$ Aklilu M, et al. This is an open-access article distributed under the terms of the Creative Commons Attribution License, which permits unrestricted use, distribution, and reproduction in any medium, provided the original author and source are credited. 
effacing (AE) because of their intimate attachment to the enterocyte and effacement of the microvillus border [7]. A chromosomal gene, $E A E C$, encodes the protein intimin, which is involved in activity [8]. AEEC which causes disease and does not produce enterotoxins or shiga toxins is referred to as enteropathogenic E. coli (EPEC).

In the study area, the detection and identification of E. coli biotypes in diarrheic lamb has not been reported. Therefore, this research was proposed to study the distribution E. coli in lamb diarrhea in and around Debrebrhan with following objectives:

1. Isolation and characterization of E. coli from diarrheic lamb feaces.

2. Identification of biotypes of E.coli isolates using standard biochemical tests.

3. Determine the association between age of lamb and occurrence of lamb diarrhea.

\section{Materials and Methods}

\section{Description of the study areas and population}

Study area: The study was conducted in and around Debrebrhan. It is city and Wereda in central Ethiopia, located in Semen Shewa Zone of the Amhara region, about 120 kilometers north east of Addis Ababa on paved high way to Dessie. The town has latitude and longitude of $9^{\circ} 41^{\prime} \mathrm{n} 39^{\circ} 32^{\prime} \mathrm{e}$ coordinates: $9^{\circ} 41^{\prime} \mathrm{n} 39^{\circ} 32^{\prime} \mathrm{e}$ and an elevation of 2,840 meters. It was an early capital of Ethiopia and afterwards, with Ankober and Angolalla, was one of the capitals of the kingdom of Shewa. There are large populations of sheep in these areas that supply meat and skin products for human consumption. Thus, the economic potential of Debre Birhan wool factory to the region in providing raw materials for wool industry is considerable.

Study population: Animals that were included in this study are cross breed lamb which are under three months old that were clinically affected with diarrhea exhibiting signs of systemic disease (poor appetite, dehydration, decreased mentation and reduced suckle reflex) and had pasty watery faeces which are found in Debre Birhan and its surrounding area.

Study design: The study was conducted in Debre Birhan veterinary clinic and the rural area around Debere Birhan from farms from November 2012 up to March 2013. Purposive type of sampling was employed, i.e., lambs showing diarrhea in the study area during the study period was used as source of sampling. Accordingly, a total of 100 lambs were sampled during the study. Fecal samples were collected from lambs that showed clinical cases of diarrhea. Approximately 50 gm of fecal material was collected from the rectum of lambs by direct digital stimulation using a disposable latex glove. Samples were placed into sterile containers and refrigerated until shipping. Samples were then transported on ice box to the institute of Biodiversity Microbiology Laboratory for sample processing. The isolate was placed in nutrient broth at $4^{\circ} \mathrm{C}$ until Biolog is conducted.

\section{Laboratory work}

Isolation of $\boldsymbol{E}$. coli: Fecal samples were inoculated on MacConkey agar (Annex-5) and incubated at $37^{\circ} \mathrm{C}$ overnight. From each plate isolated lactose fermenting colonies were inoculated on Eosin Methylene Blue (EMB) agar medium (Annex-6). For preliminary characterization colonies showing characteristic metallic sheen on EMB agar were then be picked up and considered as presumptive $E$. coli. The purified cultures of $E$. coli were stored in nutrient broth for further identification for Biolog tests, biotyping and other studies. All the isolates were stained by gram stain to determine the cell morphology and purity of the isolates. After preparing the rainbow agar, the isolated colony from nutrient broth was inoculated to confirm the colony is E.coli. Here all the colonies on the rainbow agar (Annex-7) had black or grey colors which indicate the isolate is E.coli: $\mathrm{O} 157: \mathrm{H} 7$.

Isolation of E.coli by using Biolog: After growing in EMB the purified cultures of $E$. coli were stored in nutrient broth for further identification by Biolog and sugar fermentation tests. After the E.coli was isolated on rainbow agar, it was inoculated on Bug agar (Annex 8) for further confirmation. Presumptive E. coli isolates were processed according to the procedures of the manufacturer (refer Annex 9). Briefly, a pure culture was isolated on Bug agar medium; inoculums are prepared at a specified cell density; the Biolog microplate was inoculated with the inoculums; the plate was inserted into the Biolog apparatus; the reaction pattern was entered and results were obtained from the apparatus.

Biotyping of $\boldsymbol{E}$. coli isolates: Biotyping of $E$. coli isolates was conducted based on fermentation reactions of the isolates on six sugars viz., dulcitol, raffinose, rhamnose, salicin, starch. One percent of each sugar in phenol red broth was used. For the test isolates grown in phenol red broth were inoculated into each sugar medium. Tubes were incubated at $37^{\circ} \mathrm{C}$ for seven days and readings were then recorded every 24 hours. Production of yellow color was considered as positive reaction and proper controls were kept for each of the biochemical tests performed. Isolates showing similar fermentation reaction patterns on the six sugars were considered as belonging to one biotype.

\section{Questionnaire survey}

A questionnaire was administered to lamb owners to assess the general lamb husbandry practices. Generally the questionnaire includes all practices which could have impact on the proper rearing of lambs. These include colostrums feeding, general health care, hygiene and sanitation of farms, occurrence of lamb diarrhea, preventive and control measures practiced in the farms.

\section{Data collection, management and analysis}

Data describing the diarrheal conditions suggestive of $E$. coli infection observed on sheep along with age was classified, filtered and coded using Microsoft Excel' 2007. The data were then be exported to SPSS windows version 17.0 (SPSS INC. Chicago, IL) for appropriate statistical analysis. The occurrence of $E$. coli from the total diarrheic lambs was determined by using descriptive statistics. Chi-square $\left(\chi^{2}\right)$ was used to measure the association between age and the occurrence of lamb diarrhea. Effects were reported as statistically significant if $\mathrm{P}$-value was less than $5 \%$

\section{Results}

\section{Isolation and identification of $E$. coli isolates}

For isolation of E. coli, MacConkey agar (MCA) and Eosin Methylene Blue agar (EMB) were used as differential and selective plating media. One hundred (100) fecal samples were initially screened on MCA, EMB agar and omnilog. From 100 diarrheic sheep 84 samples (84\%) were presumed to be positive for E.coli.

\section{Omnilog's identification and characterization of E. coli isolates}

The E. coli isolates were stained by Gram stain to check for the 
purity and were found to be Gram negative rods. And the isolate again cultured in to rainbow agar to confirm the isolate is E.coli (black or grey colors) and again cultured in to bug agar prepare for omnilog identification. The omnilog reading result revealed that four different species (Escherichia coli, Salmonellagp1, Serratiaodorifera ,Citrobacterfreundii ) were identified and were found to be involved in lamb diarrhea (Table 1).

\section{Biolog characterization based on assimilation result}

The equipped wotj 37-96 column panels contain carbon source for assimilation tests. Results from these tests were scored turbid metrically. The last column panel 58-60 had wells that contain 2 elements such as carbon and phosphate. These wells test for the co-utilization of various sources. (Table 2 and 3 ) shows if the read is positive on the Micro Plate, $<\mathrm{X}\rangle$, and the database result for that well is negative the printout shows $<\mathrm{X}$ - to indicate a mismatch where the database reaction is negative. If you have a negative read $\mathrm{X}$ with no brackets and database value for that well is positive and hence the well will read $\mathrm{X}+$ indicating a positive reaction in the database. At the time of a read the data is compared to the database to determine the ID. (Annex10).

\section{Distribution of diarrhea among different age groups of lambs}

The study was conducted on lambs which were less than three months of age. During the study a total of 100 diarrheic lambs were examined and from these 84 lambs (84\%) of lambs diarrhea caused by E.coli bacteria . from the whole sample which were under 1 month of age, 40 lambs $(40 \%)$,for lambs between 1-2 month of age, 35 lambs (35\%) for those 2-3 month of age 25 lambs (25\%). The age distribution of diarrheic lambs positive for E. coli was 37 lambs (43.5\%) for those which were under 1 month of age, 28 lambs (32.9\%) for those between 1-2 months of age, 20 lambs (23.6\%) for those 2-3 months of age (Table $4)$.

From the above age distribution, association between different age groups and occurrence of lamb diarrhea was calculated and showed a significant association was observed ( $P$ value $<0.05)$. Similarly association between different age groups and sheep diarrhea caused by E.coli were also calculated and showed no significant association ( $\mathrm{P}$ value $>0.05$ ). Both tests were calculated using Chi-square at $95 \%$ confidence interval.

\section{Findings of the questionnaire survey}

In this study, all the farmer followed have almost similar management system. Due to the similarity in management no statistical comparison was done for most of the factors, but some of the potential risk factors (age of lambs, colostrum feeding and hygiene of farm, treatment of cases, control measures taken) were included in the study

From the above questionnaire results the associations between lambs diarrhea and different age groups were calculated using chisquare at $95 \%$ confidence interval and the result indicates there is significant association between age group and occurrence of lamb diarrhea ( $\mathrm{P}$ value 0.03$)$

\begin{tabular}{|c|c|c|}
\hline Type of species & Number & \% of identified species \\
\hline Escherichia coli & 84 & 84 \\
\hline Salmonellagp1 & 9 & 9 \\
\hline Serratiaodorifera & 6 & 6 \\
\hline Citrobacterfreundii & 1 & 1 \\
\hline
\end{tabular}

Table 1: Biolog's identification and characterization of $E$. coli isolates.

\section{Discussion}

\section{Isolation and identification of $E$. coli isolates}

In the present study from a total of 100 diarrheic lambs $84(84 \%)$ of $E$. coli isolates were obtained from 84 rectal samples. This finding compares well the findings of Gashw M. The cultural characterization of all positive sheep E. coli revealed greenish black colony with metallic sheen in Eosine methylene blue agar, bright pink color smooth transparent colony in MacConkey agar, green color colony on rainbow agar. In Gram's staining, the morphology of the isolated bacteria exhibited Gram negative short rods arranged in single or paired.

The prevalence of $E$. coli from lambs in this study was in disagreement with reports of survey conducted in other countries. $E$. coli was isolated from $4 \%$ of ewes and lambs in the Netherlands [9], $1.4 \%$ of sheep with monthly variation of zero to $4.8 \%$ in UK [10] and $0.2 \%$ in Italy. However, a prevalence of $31 \%$ in USA and $68 \%$ from sheep flock in Australia from fecal sample were reported. These reports are much lower than the present finding. On the other hand, zero $E$. coli prevalence from sheep fecal sample was reported in Norway [11], Scotland, Ireland, Greece and United states [12]. This high prevalence in the current study may be attributed to delay in first colostrums feeding, unclean sheep houses and lack of implementation of proper preventive and control measures.

\section{Biotypes of the $E$. coli isolates}

Biochemical reactions have conventionally been used for identification of bacteria to the species level. Extensive studies of sugar fermentation reactions of bacteria have been done to introduce biochemical typing systems in epidemiological studies of bacteria $[13,14]$.

E. coli are able to ferment a variety of carbohydrate substrates, generally by converting them to glucose or to a substrate on the fermentative chain of the breakdown of glucose. The ability to ferment a given sugar of the sugars described above by a strain of $E$. coli is dependent on the strain having the requisite enzymes to convert it to glucose or to a substance on the degradative chain from glucose. It has been found that different strains of $E$. coli differ in their ability to perform these conversions. Thus, this is the basis of biotyping E. coli. These tests are easy to perform, by determining, whether a strain of $E$. coli will produce acid following growth in the presence of carbohydrates [6].

In the present study the fermentation reactions of carbohydrates by all the $84 \mathrm{E}$. coli isolates were found to be variable. Out of the $84 \mathrm{E}$. coli isolates obtained, 69 isolates were able to utilize one or more sugars while 15 isolates failed to utilize any of the sugars tested. The isolates could be grouped into various biotypes considering the fermentation reactions of six different sugars viz. dulcitol, raffinose, rhamnose, salicin, starch and sucrose. We followed the methods described by by Pandey et al. [15] and Chachra and Katoch [16] using six sugars for biotyping of $E$. coli isolates.

In this study, the most commonly occurring biotypes were Biotype I (15 isolates), III (7 isolates), II, IV, V(6), VI, VII, VIII, XI, XII, XIII, IX,(3), XIV and XV(2).The distribution of the isolates in to different biotypes indicates a wide variety in the presence of enzymes that ferments a given sugar which further implies the diverse nature of the bacteria

\section{Age association with lamb diarrhea}

In the present study the occurrence of lamb diarrhea among 
Citation: Aklilu M, Sisay T, Tefera G, Tekalign B (2013) Identification and Biotyping of Escherichia coli from Diarrheic Lambs in and Around Debre Birhan Town, Ethiopia. J Environ Anal Toxicol 3: 188. doi:10.4172/2161-0525.1000188

Page 4 of 5

\begin{tabular}{|c|c|c|c|}
\hline Biotype No. & Isolates & Total No. of isolates & Positive sugars \\
\hline $\mathbf{I}$ & $020,021,034,040,041,049,052,062,065,072,079,067,073,074,083$ & 15 & Dulicitol \\
\hline II & $006,007,027,076,048,053$ & 6 & Raffinose \\
\hline III & $015,019,060,055,054,082,085$ & 7 & Rhamnose \\
\hline IV & $035,050,047,058,061,076$ & 6 & Sucrose \\
\hline $\mathbf{V}$ & 010,039,046, & 6 & Dulicitol and rhamnose \\
\hline VI & $044,078,088$ & 3 & Raffinose and rhamnose \\
\hline VII & $029,087,0067$ & 3 & Rhamnose and salicine \\
\hline VIII & $022,066,070$ & 3 & Rhamnose and starch \\
\hline IX & $013,051,065$ & 3 & Rhamnose and sucrose \\
\hline $\mathbf{x}$ & $003,004,026,037$ & 4 & Dulicitol,raffinose and rhamnose \\
\hline $\mathbf{X I}$ & $009,069,079$ & 3 & Dulicitol,rhamnose and starch \\
\hline XII & $043,051,065$ & 3 & Raffinose, rhamnose and sucrose \\
\hline XIII & $001,025,080$ & 3 & Raffinose, salicine and sucrose \\
\hline XIV & 031,063 & 2 & Raffinose,starch and sucrose \\
\hline $\mathbf{X V}$ & 032,096 & 2 & Dulicitol, raffinose, salicine and sucrose \\
\hline
\end{tabular}

Table 2: Biotypes of $E$. coli isolates on the basis of fermentation reactions of dulcitol, raffinose, rhamnose, salicin, starch and sucrose.

\begin{tabular}{|l|l|l|l|l|l|l|l|}
\hline Age & No. of lambs & No. of E. coli +ve Samples & \%of diarrheic lambs & \% of E. coli +ve samples & OR(95\%Cl) & P-Value \\
\hline $2-3$ month & 25 & 20 & $25 \%$ & 23.6 & 32.9 & $2.13(1.23,6.78)$ \\
\hline $1-2$ month & 35 & 28 & $35 \%$ & 0.043 & 43.5 \\
\hline$<1$ month & 40 & 37 & $40 \%$ & 100 & 0.05 \\
\hline Total & 100 & 85 & 100 & 0.032 & $2.26(3.2,6.75)$ \\
\hline
\end{tabular}

Table 3: Distribution of lamb diarrhea among different age groups.

\begin{tabular}{|c|c|c|c|c|}
\hline Questionnaire Data & Category & Total Positive (\%) & OR (95\% Cl) & P-value \\
\hline \multirow{2}{*}{ Study area } & Around DebrBirhan & $53 / 65(81.5)$ & Reference & \multirow{2}{*}{$0 . \overline{626}$} \\
\hline & DebrBirhan & $31 / 35(88.6)$ & $1.13(0.59,2.18)$ & \\
\hline \multirow{3}{*}{ Age } & 2-3 month & $43 / 56(76.7)$ & Reference & \multirow{3}{*}{$\overline{0.05}$} \\
\hline & 1-2month & $26 / 28(92.8)$ & $4.46(1.67,11.89)$ & \\
\hline & Less than one month & 15/16(93.7) & $2.01(1.13,3.59)$ & \\
\hline \multirow{2}{*}{ Sex } & Male & $43 / 49(87.7)$ & Reference & \multirow{2}{*}{$0 . \overline{315}$} \\
\hline & Female & $41 / 51(80.3)$ & $1.31(0.52,3.31)$ & \\
\hline \multirow{3}{*}{ Diarrhea } & Bloody & $12 / 15(80)$ & Reference & \multirow{3}{*}{$0 . \overline{692}$} \\
\hline & Watery & $42 / 51(82.3)$ & $1.10(0.53,2.29)$ & \\
\hline & Mucoid & $30 / 34(88.2)$ & $1.47(0.69,3.14)$ & \\
\hline \multirow{2}{*}{ Sheeping facility } & Sheeping pen & $2 / 7(28.6)$ & Reference & \multirow{2}{*}{$0 . \overline{231}$} \\
\hline & Same barn & $82 / 93(88.2)$ & $1.25(0.64,2.45)$ & \\
\hline \multirow{2}{*}{ Cholustum feeding } & Yes & $83 / 99(83.8)$ & Reference & \multirow{2}{*}{0.66} \\
\hline & No & $1 / 1(100)$ & $1.31(0.59,2.90)$ & \\
\hline \multirow{2}{*}{ First feeding time } & Six and above hrs & $83 / 99(83.8)$ & Reference & \multirow{2}{*}{$\begin{array}{c}- \\
0.661\end{array}$} \\
\hline & Less than six hours & $1 / 1(100)$ & $1.93(0.61,6.15)$ & \\
\hline \multirow{2}{*}{ Sheep lost due to diarrhea } & Less than five & $56 / 71(78.9)$ & Reference & \multirow{2}{*}{0.029} \\
\hline & Five and above & $28 / 29(96.5)$ & $2.42(1.96,6.06)$ & \\
\hline \multirow{2}{*}{ Treatment for sick sheep } & Antibiotics & $34 / 48(70.8)$ & Reference & \multirow{2}{*}{0.001} \\
\hline & Tradition & $50 / 52(96.1)$ & $2.21(1.03,4.78)$ & \\
\hline \multirow{2}{*}{ Response to treatment } & Recover & $49 / 63(77.8)$ & Reference & \multirow{2}{*}{0.071} \\
\hline & Non recover & $35 / 37(94.6)$ & $1.46(0.62,3.44)$ & \\
\hline
\end{tabular}

Table 4: Description of farms based on questionnaires.

different age groups was assessed. Accordingly, young lambs (less than 1 month) of age were at a significantly high risk of being affected with diarrhea $(\mathrm{P}<0.05)$. This finding is also comparable with the result obtained from the questionnaire survey which indicated young lambs were at a significantly high risk of being affected with diarrhea than the older age groups (2-3 month age). This finding agrees well the findings of Gashaw Mersha.

The result can be associated with many factors, at younger age; delay in first colostrums feeding and unclean lamb house was associated with higher risk of morbidity. The finding that delayed colostrums intake (latter than 6 hours of age) associated with high risk of morbidity agrees with other reports. Olsson et al. [17] found that each hour of delay in colostrums ingestion in the first 12 hours of age increased the chance of a lamb becoming ill by $10 \%$. Matte et al. [18] found that $61 \%$ of colostral immunoglobulin containing $80 \mathrm{mg} / \mathrm{ml}$ of $43 \mathrm{IgG}$ is absorbed in six hours and decreases sharply thereafter. This indicates that the first six hours are the period in which maximum absorption of colostral immunoglobulin takes place [19].

The higher risk of morbidity related to delayed intake of first colostrums meal could be associated with failure of passive transfer 
Citation: Aklilu M, Sisay T, Tefera G, Tekalign B (2013) Identification and Biotyping of Escherichia coli from Diarrheic Lambs in and Around Debre Birhan Town, Ethiopia. J Environ Anal Toxicol 3: 188. doi:10.4172/2161-0525.1000188

(FPT) of cloistral immunity. The role of FPT on subsequent health and production of neonatal lambs was well documented by many researchers. There are many other similar studies which proved FPT to be a risk for lamb mortality and morbidity [20,21]. Perez et al. [22] found no difference in terms of morbidity between those ingested first colostrums before 3 hours and after three hours of age. This showed that colostrums can be still efficiently absorbed after three hours and could not contradict with the finding of the present study or other similar findings. In this study poor hygienic conditions of the observed farms may have contributed for the high occurrence of lamb diarrhea.

\section{Conclusion and Recommendations}

Lamb diarrhea is economically important health problem in sheep. During the present study isolation, identification and biotyping of $E$. coli were made from lamb diarrhea, for the first time to our knowledge. Accordingly, it was found that $84 \%$ of isolation rate of E. coli was found in lamb diarrhea in the study area. Based on their sugar fermentation abilities, isolated E. coli strains were grouped in to fifteen different biotypes. This biotyping indicated the diverse nature of the study organism in the study area.

Based on the above conclusion the following points are recommended:

The current study indicated the potential role of pathogenic E. coli in lamb diarrhea. Thus, considering the complex nature of the disease and the diverse nature of the study organism, in depth study should be done to understand and control the distribution of $E$. coli biotypes and virulent strains in lamb diarrhea in Ethiopia.

Further studies based on biotyping should be conducted to establish the association between fermentative enzymes of pathogenic $E$. coli and its pathogenicity in lamb diarrhea.

Implementation of improved lamb management practices is greatly suggested to reduce the high level of lamb diarrhea in younger lambs. Special emphasis should be given to the time of colostrums feeding, hygiene of lamb house, preventive and control measures.

\section{Authors' Contribution}

MA participated in designing of the proposal, coordinating and managing of the study, data and specimen collection, laboratory testing and drafted the article and with inputs from GT, TS, BT and AH. TS, GT, BT and AH actively participated in the study design and edition of article. Data analysis and interpretation is made by $\mathrm{AH}$. All authors read and approved the final manuscript.

\section{Acknowledgment}

We would like to deeply acknowledge College of Veterinary Medicine and Agriculture, Addis Ababa University for granting the research project. Here, we are extremely indebted to Mr. Belay Tekalegn, Dr. Genene Tefera and Dr. Tesfaye Sisay, from Mereced University, Institute of Biodiversity and Addis Ababa University for facilitation of the overall research grant, continuous support and follow up particular. We thank the Institute of Biodiversity Center at Addis Ababa particularly, Mr. Zerihun and Mis. Addis for availing their laboratory facilities. The authors also acknowledges to the owners of lambs of study areas for their collaboration in this study.

\section{References}

1. Buxton A, Fraser G (1977) Animal Microbiology. Escherichia coli, Blackwell Scientific Publications, Oxford, London, Edinburg, Melbourne 94-102.

2. Awad-Masalmeh M (2004) Virulence genes of verotoxin producing non-157 E. coli strains isolated from healthy small ruminants and cattle (in German). Monatsschrift, Wiener Tierarztliche 91: 47-55.

3. Mason RW, Corbould A (1981) Colisepticaemia of lambs. Aust Vet J 57: 458 460

4. Nazir KHMNH (2004) Molecular base of diversified Escherichia coli isolates potentiating antibiotic resistant pattern and compromising epidemiology M.S. Thesis, Department of Microbiology and hygiene, Faculty of Veterinary Science, BAU.

5. Zinnah AM, Haque MH, Islam MT, Hossain MT, Bari MR, et al. (2007) Characterization and drug sensitivity pattern of Escherichia coli isolated from different biological and environmental sources. Bangl J Vet Med 6: 13-18.

6. Crichton PB, Old DC (1982) A biotyping scheme for the subspecific discrimination of Escherichia coli. Journal of Medical Microbiology 15: 233-241.

7. Moon HW, Whipp SC, Argenzio RA, Levine MM, Giannella RA (1983) Attaching and effacing activities of rabbit and human enteropathogenic Escherichia coli in pig and rabbit intestines. Infection and Immunity 41: 1340-1351.

8. Jerse AE, Yu J, Tall BD, Kaper JB (1990) A genetic locus of enteropathogenic Escherichia coli necessary for the production of attaching and effacing lesions on tissue culture cells. Proceedings of the National Academy of Sciences 87: 7839-7943.

9. Heuvelink AE, Van de Kar NCAJ, Meis JFGM, Monnens LAH, Melchers WJG (1998) Characterization of verocytotoxin-producing Escherichia coli 0157 isolates from patients with haemolytic uraemic syndrome in Western Europe. Epidemiology and Infection 115: 1-14

10. Chapman PA, Siddons CA, Cerdan-Malo, Harkin MA (1997) A 1-year study of Escherichia coli 0157 in cattle, sheep, pigs and poultry. Epidemiol Infect 119 245-250.

11. Roger JP, Robert CC, Jeffery WB, Read SC, Rahn K, et al. (1996) Growing concerns and recent outbreaks involving non- O157:H7 serotypes of verotoxigenic Escherichia coli. Journal of Food Protection 59: 1112-1122.

12. Keenan KP, Sharpnack DD, Collins H, Formal SB, O'Brien AD (1986) Morphological evaluation of the effects of Shiga toxin and E. coli Shiga-like toxin on the rabbit intestine. Am J Pathol 125: 69-80.

13. Barr JG, Hogg GM (1979) Biotypes of Klebsiella pneumoniae and Enterobacter aerogenes characterized by differential substrate metabolism: application of the technique. J Clin Pathol 32: 935-943.

14. Krishnan C, Fitzgerald VA, Dakin SJ, Behme RJ (1987) Laboratory investigation of outbreak of haemorrhagic colitis caused by Escherichia coli O157: H7. J Clin Microbiol 25: 1043-1047.

15. Pandey PN, Thapliyal DC, Sharma SN (1979) Enterotoxigenicity of some Escherichia coli isolates. Indian Journal of Animal Research 13: 1-4

16. Chachra D, Katoch RC (1996) Prevalence of Escherichia coli and Salmonella among domestic poultry in Himachal Pradesh. Indian Journal of Poultry Science 31: 38-44.

17. Olsson SO, Viring S, Emanuelsson U, Jacobsson SO (1993) Calf disease and mortality in Swedish dairy herds. Acta vet Scand 34: 263-269.

18. Matte JJ, Girard GL, Seosne JR, Brisson GJ (1982) Absorption of colostra immunoglobulin $\mathrm{G}$ in new born dairy calf. Journal of Dairy Science 65: 17651770.

19. Foley RC, Bath DL, Dickinson FN, Tucker HA (1985) Dairy Cattle: Problems, Practices, Problems and Profits, 3rd ed. Philadelphia: Lea and Febiger 325 338.

20. Aldridge B, Garry F, Adams R (1992) Role of colostral transfer in neonata calf management: failure of acquisition of passive immunity. Compendium Continuing Education for Practicing Veterinarian 14: 265-269.

21. Le Rousie S, Klein N, Houghton S, Charleston B (2000) Use of colostrum from rotavirus- immunized cows as single feed to prevent rotavirus. Veterinary Records 147: 160-161.

22. Perez E, Noordhuizen JPTM, van Wukjkhuise LA, Stassen EN (1990) Management factors related to calf morbidity and mortality rates. Livestock Production Science 25: 79- 93 